\title{
Sartículos
}

\section{Contextualización y promoción artística dentro del marco de la nueva pintura figurativa malagueña de los 80}

\author{
Juan Carlos Martínez Manzano \\ Investigador vinculado a la Universidad de Málaga
}

RESUMEN

Este artículo trata de presentar el marco artístico de la Málaga que va desde finales de la década de los 70 a principios de los 90; partiendo de las propuestas surgidas de la generación de pintores malagueños de los 50, hasta los artistas malagueños de la nueva figuración, que colman toda la década de los 80. Mención especial a los gestores artísticos y a los espacios expositivos que acogieron las manifestaciones artísticas más importantes llevadas a cabo en este periodo, destacando las dos etapas de promotores que convergen en esta década de los 80, así como los artistas pertenecientes a la figuración malagueña.

PALABRAS CLAVE: Pintura s. XX/ Círculos artísticos/ Mecenazgo/ Málaga.

Context and Artistic Promotion into New Figurative Painting of 80 's in Málaga.

ABSTRACT

This article is about of the description the artistic framework of Malaga beginning in the late 1970s to the early 90. To beginning with the prospered from the input of the artists of the 1950 Malaga's painters generation, until the artist included in the new figuration of Málaga: those artists fill the whole 80s. Special mention to the artistic managers, and to the showrooms is also made due to they embraced the most important artistic exhibitions achieved in this period. It is also underlined the two promoters stages included the eighties, and the artists belonging to figuration of Málaga.

KEY WORDS: Painting XX th/ Artistic framework/ Sponsorship/ Málaga.

El nuevo orden cultural que se presagiaba con la transición y la labor proveniente de instituciones de reciente creación, como la joven universidad o el Ateneo, así como de otros estamentos con un dilatado arraigo en la ciudad como la "Sociedad Económica de Amigos del País", el Colegio de Arquitectos o el Museo de Bellas Artes no evitan que siga existiendo un panorama invertebrado dentro de la pintura malagueña de principios de los 80 , sin una valoración acertada de los movimientos que estaban dominando el panorama nacional. La tónica era la existencia de un gusto aburguesado, más bien decimonónico, que convivía con una generación de artistas que habían introducido algunos elementos propios de las vanguardias históricas

* MARTÍNEZ MANZANO, Juan Carlos: "Contextualización y promoción artística dentro del marco de la nueva pintura figurativa malagueña de los 80", en Boletín de Arte, $\mathrm{n}^{\circ} 29$, Departamento de Historia del Arte, Universidad de Málaga, 2008, págs. 325-347. 
hacía varías décadas. La escasa perspectiva artística directamente proporcional a una falta de formación efectiva, el escaso número de publicaciones especializadas que llegaban a manos de los artistas; así como el exiguo contacto con otros centros artísticos de mayor potencial, fueron las principales causas por las que no existió un desarrollo acorde a la infraestructura que se iba creando.

A pesar de ello, en las galerías de arte que surgen en Málaga a finales de los 60 y principios de los 70 - Picasso, Mandrágora, Malacke, Díaz- Larios, Libra, Arte Contemporáneo, Lacayí y Miramar- se percibe un cierto compromiso con la nueva corriente figurativa que en este mismo instante empieza a ser un referente en galerías de la talla de Amadís, Vandrés y Buades.

La Mandrágora será punto de referencia de la llamada "Nueva Figuración" a comienzos de los 70 en Málaga, cuando Juana de Aizpuru abre su galería en 1971 y otras como Edurne aún se encuentran inmersas en su etapa manchega. La Mandrágora comienza con dos exposiciones de artistas inmersos en esa nueva figuración, poco tiempo después de que expusieran en la galería Amadís ${ }^{1}$. Guillermo Pérez Villalta y Carlos Franco, exponen en la Mandrágora en 1972 y 1973 respectivamente. A pesar de ello, esta galería tuvo una corta trayectoria, contando con una limitada nómina de artistas hasta mediado de los 70, destacando Martínez Labrador, García Gesto, Juan Juárez, Antonio Jiménez, Begoña Garma Irarte, Carmen Moya y Mitsuo Miura, abriendo los 70 a una anquilosada política expositiva mantenida desde los estamentos oficiales y con artistas que empezaban a estar desfasados (es el caso de Eugenio Chicano, Gabriel Alberca, Francisco Hernández, Bornoy, Dámaso Ruano, Juan Béjar, Vargas Machuca ó Díaz Oliva). La que fue primera oleada de la vanguardia malagueña de los 50 y los 60 se va turnando por galerías como Lacayí, Libra, Malacke o Arte Contemporáneo durante la década de los 70 y casi a principios de los 80 , década en que algunas de estas galerías se van diluyendo hasta desaparecer o en el mejor de los casos reaparecen con renovadas intenciones, como es el caso de Malacke. Otras se reconvierten adoptando una línea convencional, obviando su apuesta inicial.

Nuevas galerías inician los 80; Arte y Belleza, Blanco y Negro, Goya, Liberty, Miramar arte, Díaz-Larios, Liceo, Miguel Ángel ó Benedito, apuestan por unos gustos tradicionales, lo que debe ser interpretado como un "desinflamiento" de la dinámica de los 70. Se suceden las exposiciones colectivas marcadas por el afán de acumular obras de diferentes tendencias sin intención de articular un discurso artístico definido, lo que terminó convirtiéndose en una constante. Esto da a entender en primer lugar un escaso compromiso de los artistas con su propia obra, buscando en estas galerías complacer los gustos de un público esporádico e indeterminado. En segundo lugar, la inexistencia de coleccionistas que motiven y despierten el interés de un mercado obsoleto y desdibujado. Este panorama desolador y repetitivo llega a

1 BOZAL V.: Pintura y escultura española del siglo XX (1939-1990), Madrid, Ed. Summa Artis, T. XXVII, pág. 578, cfr. MARCHÁN S.: "El color de los 70" en Pintura española de vanguardia (1950-1990), Madrid, Ed. Visor, 1998, págs.- 81-91. 
enquistarse de tal manera que se prescinde por completo de los escasos espacios expositivos supuestamente dirigidos a los nuevos valores y tendencias alternativas, dominados por los que se empiezan a denominar "pintores oficiales", con relativas excepciones de algunos espacios que van surgiendo durante la década.

En cierto modo el testigo de La Mandrágora lo tomarán en los 80 dos galeristas; Manuela Vilches y Esperanza Harras. Las únicas promotoras artísticas de la primera mitad de la década que provienen directamente del mundo del arte, con un amplio bagaje, a pesar de su juventud, y con ideas renovadoras pero sin el suficiente carácter como para decantarse por una línea expositiva determinada.

DE LOS ESPACIOS CONTENEDORES A LOS PRIMEROS DISCURSOS ARTISTICOS (1980-1984).

La trayectoria de Manuela Vilches desde sus comienzos parecía que podía estar a la altura de una generación de galeristas que desde principio de los 70 se iba consolidando en los focos artísticos más relevantes. Esta generación estaba formada por Juana de Aizpuru, Fefa Seiquer, Juana Mordó y Magda Belloti, como núcleo más destacado en el panorama nacional. Manuela Vilches no tuvo la consistencia necesaria ni mantuvo su apuesta de mostrar las últimas tendencias ni a los artistas con más proyección dentro del panorama local ${ }^{2}$.

En su primera exposición, optó por una colectiva de pintores malagueños de diferentes generaciones con la que tuvo una gran acogida de público, aunque no dejó de ser un escaparate más de los acostumbrados a exhibir en la Málaga cultural de principio de los 80. Intervinieron Dámaso Ruano, Manuel Barbadillo, Francisco Peinado, Chicano, Rafael Carmona, Cristóbal Toral, José María Córdoba, Mari Pepa Estrada, Diego Santos, Labrador, Juan Béjar y Francisco Hernández, así como obra gráfica de Picasso y de Bores. Una exposición representativa posiblemente de los pintores y escultores más destacado del panorama de los últimos 30 años, escogidos al azar sin criterios de estilos o de tendencias

La exposición llevada a cabo en febrero de 1981 fue prácticamente la misma que la realizada en la galería de Juana Aizpuru en 1972, con la presencia de Gerardo Delgado, Joaquín Meana, Paco Molina, Juan Ramón Sierra, Juan Juárez, Ignacio Tovar y Fernando Zóbel. La predilección por los artistas sevillanos fue una constante en las exposiciones de Manuela Vilches, inaugurando en marzo de 1981 una nueva colectiva de artistas sevillano, aunque en esta ocasión no eran tan conocidos como los anteriores. La formaron Baillo, Paloma Benítez, Fernando de Cárdenas y Manolo Castaño.

En abril de 1981 se atisba un ligero cambio en sus preferencias y encamina sus gustos hacia algunos pintores afincados en Málaga, pero sin asumir demasiados riesgos, con la muestra del pintor estadounidense, afincado en Macharaviaya, Robert ABEI. A continuación un nuevo pintor sevillano, Rafael Villanueva, que venía avalado

2 SEGURA VELASCO M. J.: “La mejor cultura”, Diario Sur, Málaga, 15-11-1981, pág. 23. 
por la consecución de premio de artes plásticas "Ciudad de Ven almádena". A partir de julio de 1981 dedica varias exposiciones a los pintores malagueños más representativos de las generaciones de los 50 y los 60 , con los que trabajará directamente a partir de este momento. Estos artistas son Elena Laverón que expone en julio de 1981 y una colectiva entre Enrique Brickmann y Francisco Peinado en septiembre de 1981, será el punto de partida de una estrecha relación profesional entre Manuela Vilches y Enrique Brickmann que culminará con la presencia en la Feria de ARCO de 1983.

En octubre de 1981 presenta la muestra de la escultora chilena Ana Aura Castro; para continuar en noviembre de 1981 con una colectiva de artistas afincados en la costa occidental con José María Córdoba, Michele Lehman, Claus Hougaard, Michael Parker, Don Parker, Birker, Pedro Escalona, Marcial Gómez, Charo García y Otero. Después de un largo paréntesis que dura más de dos años Manuela Vilches vuelve hacer acto de presencia, con una exposición dedicada a Cesar Manrique en octubre de 1984 y la apertura de una nueva galería Blanco y Negro, para posteriormente abandonar definitivamente Málaga.

Por lo que respecta a la galería Harras, fundada en junio de 1981 y dirigida por Esperanza Harras, tuvo una trayectoria expositiva más comprometida que Vilches, manteniendo en todo momento una línea más cercana a los artistas malagueños del momento, aunque también con cierta inclinación hacia las generaciones de los 50, 60 y 70 .

La primera exposición aglutina tendencias y estilos de autores dispares, con una muestra de la obra gráfica y escultórica de Alamán, Barón, Berrocal, Chillida, Chirino, Egibar, Frechlla, Gabino, Harras, Vicente Larrea, Maruna, Carlos Muela, Andrés Ángel, Oteiza, Juan A. Palomo, José Luís Sánchez y Pablo Serrano. La siguiente exposición estuvo dedicada a la polifacética artista holandesa Irene Van Vlymen, donde demostró todo su potencial artístico con óleos, acuarelas, esculturas. En agosto se llevará a cabo una exposición dedicada a los pintores malagueños Enrique Brickmann y Francisco Peinado, dentro de los actos de la "I Fiesta del Sol" patrocinada por la Diputación Provincial de Málaga. Fue una de las colaboraciones conjuntas con Manuela Vilches.

Una de las artistas más promocionada por la Galería Harras fue sin duda Machú Harras, partícipe en todas las colectivas que realizaba la galería, fue además la artista invitada por la galería en las tres ocasiones que participó en $\mathrm{ARCO}^{3}$. Machú realizó una individual en la galería en octubre de 1981, dando riendas sueltas a una amalgama de estilos y de tendencias que convirtió la exposición en todo un tratado contemporáneo de arte, desde las formas simplificadas de Brancusi, pasando por Henri Moore, Berrocal, Pistoleto etc., con un especial gusto por las obras machihembradas y desmontables. Una obra muy orgánica e íntimamente relacionada con la naturaleza tal y como en el texto del catálogo de la exposición recogía el escultor Antonio Oteiza.

En noviembre de $1981^{4}$ se realiza la exposición de Aurelio Díaz, "Buly", una

3 CAMPOY A. M.: "Comercio, cultura y espíritu creador", ABC, Madrid, 27-02-1985 y "ARCO 85" El País, Madrid, 22-02-1985.

4 BRITO C.: "Bulyrama", Diario Sur, Málaga, 18-11-1981. 
de las apuestas de la galería por la joven pintura andaluza que se venía realizando en Málaga. La exposición contó con el atractivo de una extravagante puesta en escena, con una alfombra diseñada en forma de damero blanco y negro, que se prolongaba en dirección a la calle. La alfombra parecía una extensión de la obra expuesta por Aureliano Díaz ya que coincidían los mismos tonos y colores. Este sería uno de los atractivos con los que contaba la galería, su carácter camaleónico, que más tarde adaptaría a sus exposiciones en la sala del COAM Tecla Lumbrera, adaptándose a las necesidades de la obra y a las peticiones de los artistas de transformar paredes, huecos y sombras, en un intento de mimetizar el espacio con la obra.

Para la exposición de la Navidad de 1981 la galería se dividió en cinco zonas diferentes recogiendo la obra de distintos artistas malagueños de los 50 y de los 60 . Para la ocasión en la sala denominada A se alojó la obra de los artistas Berrocal y Chicano bajo la denominación Dos malagueños en Verona; en la sala B, dibujos de Francisco Hernández con la denominación Escultura italiana contemporánea; en las salas C y D, Bornoy y Vargas Machuca Premios VI Bienal de Marbella y en la sala $\mathrm{D}$, Antonio Jiménez con Esculturas contemporáneas.

Una exposición de fotografías inaugurada el 30 de diciembre de 1981 con Carlos Barranco y José Luis Ortemín. Una conjunta con el Colegio de Arquitectos de Málaga de los pintores Rafael Hidalgo de Caviades y Ángel del Río, inaugurada con un concierto a cargo de Virginia Luque y Christian Balde. En enero y febrero de 1982 la exposición del artista norteamericano Jerry Sherry. En abril de 1982 le llega el turno al joven malagueño Marcelo Arce. Elvira Cue en mayo de 1982 y Felipe Orlando en octubre de 1982, con una exposición que denominó Orbita, organizada conjuntamente con el colectivo $7 / 10$.

En noviembre de 1982 se inaugura una exposición del artista norteamericano Robert Harvey, con una larga trayectoria de exposiciones en la costa; al que sigue otro joven artista malagueño Juan Carlos Blanca, dentro del programa de oportunidades que Esperanza Harras ofrece a los jóvenes pintores malagueños. En diciembre de 1982, de nuevo la típica muestra colectiva de navidad con Fernando Teixidor, Margaret Harding, Robert Harding, Amal y Perry Oliver, acabando el año con una individual de Fernando Teixidor.

En 1983 la galería Harras acude a la feria internacional de ARCO con los artistas Machú y Antonio Jiménez y en mayo de 1983 será una de las tres sedes de la exposición Vidas Modernas, muestra internacional de arte organizada por Joaquín de Molina y Gabriel Padilla. A partir de este momento y hasta diciembre de 1983 la galería Harras se ve obligada a cerrar ${ }^{5}$ por falta de solvencia, reapareciendo con una exposición dedicada a Barbadillo.

Otros espacios abren sus puertas a principios de la década con el ánimo de poder contribuir a la renovación de las artes plásticas y servir a las nuevas genera-

5 En enero de 1983 se publica en la prensa -vid. Diario Sur, 24-01-1983- una carta abierta donde se denuncia la situación de la infraestructura artística de la ciudad, exponiendo como ejemplo la precariedad de la Galería Harras. La carta será firmada por un amplio colectivo de galeristas, críticos y artistas. 
ciones para que expongan sus primeras obras y darse a conocer dentro de lo que parecía un coto cerrado a los pintores de las generaciones anteriores. La lista es extensa, aunque los que tuvieron una cierta relevancia fueron Arkadia, Arcorac, Yunque, la sala de arte del Ateneo de Málaga, la sala del COAM y otras salas de carácter más alternativo como Arte-bar, Pámpano, Casablanca y Terral.

En la Casa de la Cultura se dan a conocer artistas con escasa proyección mediática y que mantienen una línea al margen de los ya consagrados. Podríamos citar a José Carlos Ambrosio que expuso en julio de 1980, sin una línea de actuación definida, pero que apuntaba hacía una especie de obra cinética que recordaba las instalaciones de Agam, aunque en otros momentos se inclinara por crear grandes máquinas al estilo de Jean Tinguely. En enero de 1981 expone Marcelo Arce con una visión cinematográfica del realismo fantástico, una de las vías figurativas empleadas por los pintores malagueños de los primeros años 80. Entre marzo de 1981 y febrero de 1982 exponen artistas prácticamente desconocidos: Enrique Vilches, Ricardo Torrente, Juan Ruiz Sánchez, Enrique Páez, Francisco Navas, Francisco Peña, Hierrezuelo, Isabel León, José Pereiro, Antonio Portero e Ignacio Navarro. En febrero de 1982 un joven José Antonio Cepeda sorprende con su serie blanco y negro un claro exponente del movimiento Support Surface, que mantiene durante los 70 la línea informalista de los artistas europeos. Escaso interés durante la primera mitad de la década de los 80 fueron las exposiciones de Toré Blanca, Antonio López Portero, José Hidalgo, Antonia Soler y Esther Sánchez.

La sala de exposiciones de la Caja de Ahorro de Antequera, creada en 1977 contó con la dirección de Alfonso Canales, que mantuvo la galería a disposición de jóvenes artistas que aun seguían experimentando con pasadas experiencias abstraccionistas y elementos más propios de las vanguardias históricas. En este apartado destacaron las exposiciones de Adolfo Santarem, Díaz Del, Juan Carlos Blanca, y Salvador Fajardo. En enero de 1983 las exposiciones de la CAA, parecen tomar cierto aliento con la presencia de José Aguilera, uno de los pintores más reconocidos en los 70 y que a principio de los 80 parece mantenerse vigente. A la exposición de Aguilera, le siguieron algunas exposiciones de pintores que posteriormente serán piezas fundamentales de la pintura de los 80 , es el caso de Rafael Alvarado y Enrique Queipo. Otros pintores jóvenes que expusieron en estos primeros años de la década fueron: Lope Martínez Alario, José Antonio Cepeda, Juan Pinilla, Miguel Zoi, Benito Lozano y la exposición colectiva llevada a cabo en junio de 1985 titulada "Pintando en Málaga"6 con Alvarado, Juan Crossa, Cornejo y Paco Rojos.

Otro de los espacios creados al inicio de la década de los 80 será la sala de arte del Ateneo. Sus pequeñas dimensiones no fueron impedimento para que expusieran los más destacados artistas de la llamada "Nueva Figuración", fruto del trabajo de Andrés García Cubo.

En el periodo que va desde julio de 1980 hasta julio de 1983 la nómina de

6 Vid. Diario Sur, 6-6-1985. 
artistas es larga por lo que haré mención solo de aquellos más significativos. En julio de 1980 expone Rafael Alvarado, a pesar de su juventud era uno de los pintores que ya contaban para los galeristas malagueños del momento. En ese mismo mes llega la exposición de Joaquín de Molina, uno de los artistas que en 1979 había participado en la exposición "Nueva Figuración" celebrada en el museo de Bellas Artes; al que le sigue Gabriel Padilla7, otros de los pintores que expusieron en aquella exposición del Bellas Artes. En octubre de 1982 expone el colectivo Agustín Parejo School con una serie de obras en collage tituladas Agit Pop y en noviembre Chema Tato. En enero de 1983 se celebra 1.000.000 de pavos ${ }^{8}$, donde se da cita un elenco de artistas tanto locales como nacionales relacionados directamente con el movimiento figurativo de los 70 y de los 80., destacando: Díaz Pardo, Plácido Romero, Chema Tato, Bola Barrionuevo, Daniel Muriel, Agustín Parejo School., Queipo, Octavio Colis, Pérez Villalta, Seguiri, Ouka Lele, Chema Lumbreras, Antonio Herrainz, Martín Begue, Parody, Isabel Garnelo, Patricia Gadea, Rafael Pérez Estrada, Eduardo Guille, Fons Bada, Herminio Molero, García Alix, El Hortelano, Dorotea Von Alba, José María Báez, Miguel A. Albarracín, Carlos Durán, Palín Casares, Jaime Gorospe, José Morea, Andrés García Cubo, Elena Blasco y El Ojo. Las siguientes exposiciones serán una alternancia entre los pintores malagueños con más proyección dentro de la figuración y los pintores de la "Generación del Arco del Poniente Gaditano", compuesta por Enrique Naya, Juan Carrero, Evaristo Belloti, Pérez Villalta y Chema Cobos, consagrados en Madrid y artífices de la que se hizo llamar "Neofiguración Madrileña". Se suceden Pepe Seguiri, Bola Barrionuevo, Carlos Durán, Alfonso Albacete, Costus, Pérez Villalta ${ }^{9}$, Fons Bada y Elena Blasco. Hacer mención de las exposiciones de Pablo Pérez Mínguez bajo el título Vírgenes y Mártires, celebrada en julio de 1983 y la colectiva integrada por Costus, Olvido Gara, Fanny Mcnamara, Carlos Berlanga, Miguel Ordóñez y Tesa, con fotografías de García Alix.

La sala de arte de la Diputación de Málaga, dirigida desde 1971 por Miguel Alcobendas, completó tres etapas expositivas bien diferenciadas. La primera va de 1971 a 1981, con exposiciones de artistas de las generaciones de los 50 y $60^{10}$. El segundo periodo comprende desde 1980 a $1982^{11}$, una etapa donde tiene cierto protagonismo la generación figurativa de finales de los 70 . Esto no llegó a ser del todo cierto, ya que en esta segunda etapa continuó con exposiciones de artistas de gene-

\footnotetext{
7 Sobre esta exposición vid. CAMACHO, R.: "Gabriel Padilla, en la sala del ateneo", Diario Sur, Málaga, 23-11-1980. 8 Diario Sur- 9-1-1983.

9 La exposición de Pérez Villalta denominada "Oro, Copas, Espadas y Bastos" se considera una de las exposiciones más importantes llevadas a cabo en Málaga durante la primera mitad de la década. Vid. Diario Sur, 17-6-1983.

10 Con estos artistas ya había trabajado en un documental producido y dirigido por el mismo sobre la obra de la llamada Vanguardia Malagueña de los 50, titulado "La pintura de vanguardia en Málaga", de 1970.

11 Para tener un conocimiento completo de las exposiciones de este periodo vid. ALCOBENDAS, M.: La pintura contemporánea en Málaga: Sala de exposiciones de la Diputación Provincial de Málaga, Málaga, Ed. Diputación Provincial, Servicio de Publicaciones, 1982.
} 
raciones pasadas como es el caso de Bornoy, que expuso en octubre de 1980; Díaz Oliva, en noviembre de 1980; Barbadillo, en diciembre de 1980 y Aguilera, en febrero de 1981. En marzo de 1981 comienzan las exposiciones más acorde con la nueva generación que se da a conocer a finales de los $70^{12}$, comenzando con la poesía visual de Lorenzo Saval, al que le sigue la colectiva del grupo "El Grifo" con los jóvenes Santarem, Ambrosio y Cepeda, y una exposición de Diego Santos en mayo de 1981. A esta exposición le sigue una extraña e innecesaria colectiva bajo la denominación La pintura contemporánea en Málaga" con autores dispares en todos los sentidos y que habían expuestos hacía pocos meses. Esta exposición la conformaban: Aguilera, Alberca, Béjar, Bonilla, Bornoy, Díaz Oliva, Hernández, Hildalgo, Lindell, Maruna, Ruano, Santos y Valdés.

Este periodo es más bien de transición, ya que a partir de noviembre de 1981 se suceden una serie de exposiciones de los artistas que inician los 80 , es el caso de las exposiciones de Díaz Del, noviembre de 1981; Antonio Herraiz, noviembre de 1981; Paco Aguilar, febrero de 1982; Gabriel Padilla, marzo de 1982; Pedro Saavedra, en abril de 1982; José María Prieto, en mayo del 1982; Nuño-Ruiz, en junio de 1982 y Manuel Álvarez en junio de 1982, con lo que finaliza una segunda época que no cubrió las expectativas anunciadas en un principio, dejando fuera a muchos nombres que por aquel tiempo comenzaban sus primeras exposiciones.

En la tercera época, recogida en otro libro publicado en $1984^{13}$, el cambio fue radical y apostó por mostrar otras disciplinas artísticas, en su gran mayoría fotografías, con algo de alfarería, tejidos y una sola exposición de pintura que correspondió nuevamente a José Aguilera. Sin duda la exposición más significativa fue la de Miguel Trillo en marzo de 1984, uno de los fotógrafos más importante de la denominada "Movida Madrileña".

En abril de 1980 se pone en funcionamiento la sala de exposiciones del Colegio de Arquitectos ${ }^{14}$ donde destacaremos de momento dos exposiciones; una exposición de escultura con Elena Laverón y Machú Harras y una muestra de la obra gráfica del colectivo 7/10 dentro de una estructura neumática obra del arquitecto José Miguel Prada Poole.

Las salas temporales del Museo de Bellas Artes se reservaron para grandes exposiciones antológicas, con la clara excepción de la exposición colectiva De la nueva figuración ${ }^{15}$ inaugurada en octubre de 1979, donde estuvieron presentes algunos de los artistas de la nueva figuración más relevantes del panorama andaluz, dando a conocer algunos nuevos valores en ciernes como Víctor Carro, Antonio Belmonte, Carlos Casariego, Eloy Cuadra, José Díaz Pardo, Andrés García Cubo, Amelia Jiménez, Santiago Lluch, Joaquín de Molina, Rafael Núñez, Gabriel Padilla,

12 Ibídem.

13 ALCOBEndAS, M.: Los otros lenguajes de Málaga, Málaga, Ed. CEDMA, 1994.

14 La noticia de la apertura de la nueva sala de exposiciones del Colegio de Arquitectos es recogida en Diario Sur, el 26-6-1980.

15 CAMACHO R.: "Veinte pintores jóvenes en Bellas Artes", Diario Sur, Málaga, 25-10-1979. 
Rafael Pérez Estrada, Antonio Posada, José María Prieto, Santiago Serrano, Pablo Sycet, Antonio Tilman, Jan Tverin, Vargas y The Yves Factory. La gran mayoría de estos artistas estaban directamente relacionados con el entorno de la pintura andaluza de comienzos de los 80 , por lo que puede representar una escuela de pintores figurativos propiamente de Andalucía y el inicio de diversos focos localistas figurativos.

Desde finales de los 70, la Universidad de Málaga, a través de la Secretaria de Extensión Universitaria, configura un programa expositivo en el Museo de Bellas Artes, comisariado por Isidoro Coloma Martín recogiendo un amplio espectro de artistas y colectivos que, en cierto modo, han polarizado el arte de los últimos treinta años, intercalando en su tarea expositiva artistas locales de diversa consideración. De estas exposiciones cabe destacar la llevada a cabo en 1977 con obras del colectivo EI Paso; Alberto Sánchez en febrero de 1981 o la de Henri Moore, en marzo de 1982 con la particularidad de su ubicación en la galería Miguel Ángel. Otras exposiciones claves de este periodo fueron las de Joan Miró en junio de 1981, "Málaga y Picasso" de noviembre de 1981; así como "Arte español contemporáneo" de 1981 con toda una generación de artistas representativos de la década de los 60 y los 70, destacando Gerardo Rueda, Eusebio Sempere, Farreras, Clavé, Cuixart... y una larga lista de artistas de los últimos 30 años.

La Academia Arkadia y la Sociedad Arcorac que nacen al amparo de sendas iniciativas privadas, se sitúan en un entorno acomodado y con unas instalaciones demasiado costosas para sus pretensiones. La Academia Arkadia, creada en mayo de $1983^{16}$, por iniciativa de Concha Escassi y Violeta Cárdena emprenden una efímera aventura como galeristas, destacando la exposición El mar en tus ojos, una colectiva formada por artistas malagueños, plenamente integrados en el organigrama de los 80: Ayuso, Carlos Durán, Herrainz, Chema Lumbreras, Joaquín de Molina, Daniel Muriel, Gabriel Padilla, Agustín Parejo School, Diego Santos, Pepe Seguiri y Tecla Lumbreras. La importancia que tuvo esta exposición radicó en ser la primera vez que el elenco de la generación figurativa malagueña de finales de los 70 y principios de los 80 coincide al completo en una exposición, a excepción de Bola Barrionuevo y Chema Tato.

Por su parte Arcorac se crea en noviembre de 1981 por iniciativa de Juan Carlos Narbona Gemar, José Leandro Quero y Antonio Bustamante, La única muestra digna de mención fue una colectiva de artistas de la Axarquía que tuvo lugar en enero de 1982. En aquella ocasión participaron Antonio Hidalgo, Antonio Valdés, Jurado Lorca, Lope Martínez Alario, Fortes, Pepe Bonilla, Gallardo Gaspar y Sotomayor, con algunos de los exponentes del movimiento figurativo propio de Vélez Málaga.

Los dos espacios expositivos con más entidad dentro de los denominados "pub-galerías" serán Terral, y Casablanca. Terral se inaugura en mayo de 1984.por iniciativa de la controvertida Pilar Chamorro ${ }^{17}$. Comprometida con la nueva figuración

16 SAINZ DE LOS TERREROS J.: “Un proyecto común. La academia Arkadia”, Diario Sur, 26-5-1983.

17 En la revista El Observador, $n^{\circ} 10$, se publicó una interesante entrevista a Pilar Chamorro donde se deja claro los motivos y las directrices de Terral, así como se analiza la personalidad de esta promotora artística, 
y con la vanguardia artística del momento, Terral tuvo una dilatada existencia, adentrándose en los 90. De las exposiciones llevadas a cabo por Pilar Chamorro en Terral destacar la celebrada en mayo de 1985 con obra gráfica de Paco Aguilar, Barbadillo, Béjar, Brinkmann, Casadama, Farias, Gordillo, José Hernández, Pijoán, Miura, Peinado, Ruano, Saura, Pepe Seguí, Antonio Serrano y Tapie. Las siguientes exposiciones de Terral si estuvieron más a la altura de lo pronosticado por Pilar Chamorro, con las muestras de Antonio Barba, en septiembre de 1984, la exposición de Buly, Hematemesis, en septiembre de 1984; la colección de camisetas diseñadas por el colectivo Agustín Parejo School bajo el lema viste un poema, pintadas a mano con diferentes motivos y referencias. En abril de 1985 expone Melguizo y en mayo Lope Martínez Alario. En noviembre de 1985 Enrique Carmona para terminar la primera mitad de los 80 con una exposición de Chema Lumbreras, Tendor y Paco Aguilar.

Del pub Casablanca hacer mención de una exposición celebrada en 198418, donde participaron once pintores malagueños adscritos a las dos primeras generaciones de pintores figurativos que se dieron cita en Málaga en los 80. Estos fueron: Carlos Durán, Alfonso Serrano, Andrés García Cubo, Tecla Lumbreras, Chema Lumbreras, Pepe Seguiri, Mario Martín, Gabriel Padilla, Joaquín de Molina, Elena Bravo y Plácido Romero. La novedad que presentaba esta muestra era la intervención de los artistas en vivo, de cara al público. Esta especie de performance fue idea de Joaquín de Molina, contando con el apoyo de estos diez pintores. En este punto se fundamenta otros de los cambios de actitud cercanos a la mitad de la década y que sería premonitorio de lo que estaba por llegar.

Por lo que respecta al colectivo Palmo ${ }^{19}$, la nueva figuración se vio favorecida por el programa de concursos de pintura llevados a cabo durante la primera mitad de la década. En septiembre de 1980 se realiza la primera muestra con alumnos del taller; Alvarado, Hierrezuelo, Francisco Sánchez, María Antonio y José Ambrosio y en septiembre de $1981^{20}$. La primera muestra con los pintores que fueron seleccionados en el primer certamen de pintura joven con: Lope Martínez Alario, Ruiz-Juan, Gabriel Padilla, Santarem, Sebastián Camps y Antonio Herraiz. El segundo certamen de pintura joven se celebra en julio de 1982; en esta ocasión los seleccionados fueron Rafael Alvarado, José Antonio Villanueva, Lope Martínez Alario, Francisco Mayorga, Enrique Queipo y Ganfornina, siendo el ganador de la muestra Lope Díaz Alario. En abril de 1984 se convoca el tercer certamen de pintura joven, cuyo fallo se produce el 2 de mayo de 1985. El galardonado fue Chema Lumbreras, habiendo que-

pieza fundamental dentro del organigrama de los artistas figurativos malagueños de la segunda mitad de la década de los 80. Vid. GONZALEZ F.: "Pilar Chamorro: Los organismos públicos tiran el dinero", El Observador $n^{\circ} 10$, Málaga, 1987.

18 ESCUDERO C.: "Once pintores malagueños realizaron una muestra en vivo de su arte en el pub Casablanca", Diario Sur, Málaga, 23-7-1984; GONZALEZ C.: "Experiencia moderna con once pintores", El Sol de España, Málaga, 23-7-1984.

19 Vid. CASTAÑO E.: "La experiencia artística y personal del colectivo Palmo",

20 CONTRERAS S.: "Jóvenes pintores malagueños en el colectivo Palmo”, Diario Sur, Málaga, 19-9-1981. 
dado finalistas Francisco Pérez e Isabel Jaramillo. En junio de 1985 tiene lugar el cuarto certamen, premiando la obra de Isabel Garnelo, con la consiguiente exposición anunciada para el 24 de septiembre de $1985^{21}$.

PROMOTORES ARTISTICOS Y ESPACIOS EXPOSITIVOS EN LA SEGUNDA MITAD DE LA DÉCADA. LA INICIACIÓN DE LOS DISCURSOS ARTISTICOS (19851989).

A excepción de Andrés García Cubo, cuya labor se inicia a principio de los 80, en la sala de arte del Ateneo de Málaga, el resto de los nuevos promotores que contactan con la nueva pintura figurativa malagueña serán un grupo de jóvenes, en su mayoría historiadores del arte que desde diversos ámbitos coinciden en Málaga durante la segunda mitad de la década de los 80 . Los nombres de Tecla Lumbreras, Joaquín Abenza, Pedro Pizarro, Carmen de Julián, García Agüero, Carmen Romero y Pilar Chamorro, tomarán el testigo de aquellos otros promotores de la primera mitad de los 80 , en su gran mayoría comerciantes de otras ramas, que desembocan en el mundo del arte de manera fortuita; es el caso de Miguel Alcobendas, Alfonso Canales, Juan Carlos Garbona Gemar, José Leandro Quero Ayllón, ó Miguel Ángel Piedrola.

Con respecto a la crítica y la edición de revistas especializadas, el panorama da un vuelco espectacular, comenzando a gestarse una joven generación de críticos de arte, inexistente por completo durante la primera mitad de la década, a través de revistas como Bulevar, El observador o Imágenes alteradas, a lo que hay que añadir una prensa más comprometida con el arte de vanguardia, comenzando a dedicar suplementos semanales y páginas diarias a los artistas y a las galerías.

Uno de los primeros que introduce en sus comentarios elementos propios de una crítica de arte puramente objetiva y sin recurrir a una retórica exacerbada, quizás sea Guillermo Busutil, con un estilo directo y agudo que recuerda la crítica que se venía ejerciendo en las principales revistas nacionales. Críticos como José Luis Brea, Rosa Olivares, Juan Antonio Ramírez, Mar Villaespesa o Kevin Power ejercían una influencia decisiva dentro del panorama artístico, preferentemente a través de las revistas Lápiz y Figuras.

Con la creación de Bulevar, El Observador e Imágenes Alteradas se va introduciendo un joven plantel de críticos y comentaristas procedentes del ámbito universitario que muestran su interés por un grupo de galerías, no solo malagueñas sino también de algunas ciudades andaluzas como son las galerías de Magda Belloti, Sanrandunga, Aizpuru y Rafael Ortiz. Empieza a producirse una cierta complicidad entre algunas galerías y los jóvenes críticos, aunque esta relación no llegará ser tan estrecha como la que existió entre la galería sevillana de La maquina española y la revista Figuras. Las revistas Bulevar y El Observador, tenían cierta predilección por las galerías de Pedro Pizarro, la sala del Colegio de Arquitectos y Carmen de Julián.

21 Vid. MAYORGA J.: "La pintura de Isabel Garnelo", Diario Sur, Málaga, 29-9-1985. 
Este acercamiento entre las nuevas galerías de arte y las también nuevas revistas ha sido más importante de lo que parece a simple vista, ya que marcarían la frontera de calidad entre la gran mayoría de los espacios expositivos y estas pocas salas de arte comprometidas con el arte más inmediato de los jóvenes artistas figurativos. El ejemplo mas claro de esta afinidad se pudo vislumbrar entre Bulevar y la galería de Pedro Pizarro, por algunas circunstancias que fueron evolucionando con el paso del tiempo; la utilización de las instalaciones de la galería para la presentación de algunos números de la revista; las reseñas de todas las exposiciones de Pedro Pizarro, así como numerosas entrevistas dedicadas a la figura del galerista.

Hubieron algunos antecedentes de revistas dedicadas a las artes plásticas durante el primer lustro de la década, es el caso de Ciencias y letras, que publicó su primer número en marzo de 1981 con la iniciativa del Colegio Oficial de Doctores y Licenciados del distrito universitario de Málaga y dirigida por Rosario del Pino. Pero fue Bulevar la primera revista, si exceptuamos Boletín de Arte, dedicada casi en exclusiva a las artes plásticas con un plantel de jóvenes críticos como: Álvaro García, José Antonio Mesa Toré, Alfredo Taján, y Esther Morillas entre otros. Por lo que respecta a la revista El observador se ceñía más a las características de un magazín, con un amplio apartado denominado "Existencias" dedicado a exposiciones y entrevistas . Dentro de El observador los colaboradores eran fijos, destacando en la sección dedicada a exposiciones las colaboraciones de Francisco González y Angélica Gómez.

En prensa también se produjeron grandes novedades, que vinieron de la mano de algunos suplementos culturales como el de Diario 16, publicado en un principio desde Sevilla, lo que no impidió un seguimiento continuo de las novedades que se estaban produciendo en Málaga. De entre sus colaboradores destacar a Kevin Power, Eduardo Herreros, Francisco Rodríguez, Héctor Perea y Miguel Fernández Cid.

A partir de 1986 nacen varios diarios locales que apuestan de manera decidida por introducir en sus contenidos secciones de arte de forma regular. Hay que destacar El Sol del Mediterráneo que desde 1988 a 1990 dedicó en su dominical una sección de cultura que contó con una extensa plantilla de jóvenes escritores, destacando; Isabel Pérez Montalbán, Chelo Navas, Laura Smith, Manuel Fernández y Javier Cuenca. En todos ellos se percibe una mayor especialización en los contenidos, con un conocimiento y un acercamiento al arte del momento, pero como ocurría con Bulevar le dedican una atención especial a la galería de Pedro Pizarro, con un seguimiento constante y puntual que distaba mucho de la atención prestada a las otras dos galerías - Carmen de Julián y Colegio de Arquitectos- que también apostaron por este tipo de arte. Otro de los diarios que se crea a partir de 1987 es la Gaceta de Málaga con Guillermo Busutil y Gonzalo Fausto.

Algunos de los galeristas que comienzan sus actividades durante la segunda mitad de la década están determinados por un conocimiento del contexto artístico globalizado. Se vivía con interés los últimos momentos de uno de los acontecimientos más prolificos dentro de los movimientos culturales de los últimos años, la llamada "Movida" que ya había penetrado en Málaga de la mano de García Cubo. La sala de exposicio- 
nes del Colegio de Arquitecto (COAM) dirigida por Tecla Lumbreras captó su esencia y no solo atrajo a su galería a los denominados "Artistas Alternativos" de la movida Ceesepe, Pérez Mínguez, El Hortelano, Trillo, Nazario, Ouka Lele, Carlos Berlanga sino que se liberó de la carga de intelectualidad del informalismo aún imperante y de la corriente figurativa de los 70, que siguieron la estela de Luis Gordillo, Kitaj, Hockney y Katz, por una nueva línea que se denominó de la "Figuración Crítica".

El enriquecimiento del panorama expositivo se debe también en gran medida a la notable información que estos nuevos galeristas tenían de algunos de los artistas figurativos europeos más importantes del momento. De ello dan debida cuenta las exposiciones de Nino Longobardi, Milan Kunc, Jiri Georg Dokopuil y Markus Oehelen, pertenecientes a una corriente del realismo crítico-social que procede especialmente de los países del este europeo y de grupos como Heftigr Malerei y Mülheimer Freinheit.

La galería de Pedro Pizarro inicia su actividad en Alhaurín el Grande en 1986 , con una colectiva dedicada a la figura de San Sebastián. Con esta primera exposición muestra la línea y los artistas con los que trabajará desde este momento hasta la conclusión de sus dos periodos. Estos artistas son Rafael Agredano, APS, Miguel Ángel Abarracan, Manuel Caballero, Andrés Cabrera, Patricio Cabrera, Salomé del Campo, Curro Casillas, José Luís Díaz Pardo, Curro González, José María Larrondo, Benito Lozano, Joaquín de Molina, Moisés Moreno, Guillermo Paneque, Antonia Peña, Plácido Romero, Pedro Simón, Eduardo Solera, Antonio Sosa, Ignacio Tovar y Alejandro María del Verbo.

La gran mayoría de estos pintores son sevillanos o están muy relacionados con el entorno hispalense, en concreto: Andrés Cabrera, Patricio Cabrera, Curro González, Antonio Sosa, Ignacio Tovar, Curro Casillas, Salomé del Campo, Guillermo Paneke y Moisés Moreno son pintores nacidos en Sevilla entre 1954 y 1962. Otros como Manuel Caballero o Rafael Agredano muy relacionados con el ambiente artístico sevillano a través de la galería La Máquina Española y la revista Figuras. El resto son artistas malagueños: APS, Benito Lozano, José Luis Díaz Pardo, Plácido Romero y Joaquín de Molina.

Con el colectivo APS22 Pedro Pizarro lleva a cabo una exposición en junio de 1986, con el título Consigna; además de participar en esta primera etapa en una de las exposiciones colectivas más significativa de su periplo; Co-mercia ${ }^{23}$, realizada en junio de 1986, con la participación de: APS, José Luis Santana, Diego Santos, Cristina Romero, Moisés Moreno, Benito Lozano, José María Larrondo, José Antonio López Cuenca, José María Córdoba, Salomé del Campo, José Miguel Jiménez, Andrés Cabrera y Manuel Caballero. La idea de proponer una temática amplia, ambigua e inconcreta despertó el imaginario artístico de todos los artistas invitados, lo que enriqueció el resultado final, sin dejar de ser un juego, una experiencia lúdica que provocaba una efectiva distensión y que contagiaba al público.

22 GARCIA P.: "Continúa la exposición de APS en la galería de Pedro Pizarro", Diario Sur, Málaga, 17-7-1986. 23 GARCIA P.: "Co-mercia, exposición colectiva en el primer aniversario de la Galería Pizarro", Diario Sur, Málaga, 18-6-1987. 


\section{Q: artículos Juan Carlos Martinez Manzano}

De estas dos colectivas se pueden extraer algunas conclusiones, la más importante, sin duda, la apuesta de Pedro Pizarro por un grupo de artistas malagueños donde tienen cabida muchos de los miembros de la generación figurativa de finales de los 70, es el caso de Carlos Durán, Gabriel Padilla, Chema Tato, Daniel Muriel y Pepe Seguiri, a los que posteriormente se les unen los malagueños de la siguiente generación como Chema Lumbreras, Isabel Garnelo y Plácido Romero. Otra de las exposiciones conjuntas que muestra interés por los pintores malagueños de la última hornada figurativa será la celebrada en diciembre de 1986 donde participaron Chema Lumbreras, Isabel Garnelo y José Luis Castellanos ${ }^{24}$.

La colaboración con Diego Santos da sus frutos en dos exposiciones; en febrero de 1987 y en marzo de 1989, coincidiendo con las dos etapas en que se divide la labor expositiva de Pedro Pizarro. Otros artistas malagueños que expusieron con Pizarro en su primera etapa fueron: Sebastián Camps, en noviembre de 1987; Juan Antonio López Cuenca, en febrero de 1988 y Carlos Canal, en abril de 1988, para finalizar su estancia en Alhaurín con dos colectivas: ¡Málaga virgen!, la unión del arte y la empresa ${ }^{25}$ y Nueve artistas 26 .

En enero de 1989, Pizarro inaugura su galería de Málaga con una colectiva compuesta por: Patricio Cabrera, Pepe Espaliú, Bernardo Friza, Curro González, Pedro G. Romero, Begoña Goyenetxea, Milan Kunc, Rogelio López Cuenca, Eva Lootz, Markus Oehelen, Pedro Proenca y Cindy Shermann ${ }^{27}$. Una autentica torre de babel de artistas que van resumiendo parte de las tendencias pictóricas de la figuración de los 80. Durante la temporada 88-89 destacar las siguientes exposiciones: Diego Santos, con la exposición denominada Pieles en marzo de 1989; la obra del artista brasileño, afincado en Francia, Roberto Cabot, en abril de 1989; el joven artista sevillano Pedro G. Romero, en mayo de 1989 y Rogelio López Cuenca, en junio de 1989. Mención especial merece la participación de la Galería Pizarro en la I Semana de Arte Contemporáneo TRIACA, celebrada en Málaga, en junio de 1989, en el Centro de Exposiciones Sur, bajo la coordinación de Guillermo Busutil28. La galería se presenta a esta cita con la obra de Diego Santos, Antonio Yesa, Odile Ruiz, Nino Longobardi y Markus Ohelen.

La temporada 89-90 es la del asentamiento de la galería en la ciudad y punto de referencia cultural en muchos aspectos; desde la difusión de nuevos valores provenientes de los más diversos campos artísticos, siendo el escaparate de una nueva generación artística, la de los 90, con un acercamiento a la vanguardia conceptual malagueña con artistas como Jorge Dragón, Joaquín Ivars, Rogelio López Cuenca, Encarni Lozano y Odile Ruiz, que clausuran definitivamente la figuración de finales de los 70 y casi toda la década de los 80 . El único autor procedente de la figuración

24 GARCIA P.: "Exposición conjunta de Chema Lumbreras, Isabel Garnelo y José Luis Castellanos", Diario Sur, Málaga, 3-12-1986.

25 Vid. La Gaceta de Málaga, 7-12-1986.

26 GARCÍA P.: "Nueve artistas exponen en la galería Pizarro", Diario Sur, Málaga, 7-9-1988.

27 MAYORGA J.: "Galería Pedro Pizarro, nuevo espacio cultural para la ciudad", Diario Sur, 16-2-1989.

28 SMITH L.: "Triaca, un lugar para crear y mostrar", El Sol Del Mediterráneo, Málaga, 20-6-1989. 
de los 80 que expone durante esta temporada en la galería Pizarro será José Luís Bola Barrionuevo, en febrero de 1990, con la exposición El limite entre la tierra y el mar29, así como la muestra Obras recientes de Chema Cobos en mayo de 1990.

Tecla Lumbreras se inicia como promotora artística en el taller de grabado del colectivo 7/10, Más adelante José Ignacio Díaz Pardo y Maria Eugenia Candau, miembros pertenecientes a la comisión de cultura de la sala de exposiciones del Colegio de Arquitectos, le ofrecen dirigir la sala de arte a lo que accede en 1984, prolongándose su actividad hasta noviembre de $1993^{30}$.

Contó para su largo proyecto con los artistas malagueños de la generación de los 80 más influyentes; Diego Santos, Carlos Durán, Bola Barrionuevo, Seguiri, Chema Tato, Padilla, Plácido Romero y un largo etc. que supo combinar con otros artistas como Ouka Lele, Trillo, Mínguez, El Hortelano, Pérez Villalta o Rafael Agredano. El cambio fue inmediato, de exposiciones meramente convencionales durante el primer periodo de la sala de arte del COAM se pasó a todo tipo de manifestaciones artísticas y eventos sociales que abrió todo un abanico de posibilidades, combinando en multitud de ocasiones el espacio interior de la galería con el aprovechamiento del gran espacio exterior del recinto del COAM.

Las primeras exposiciones llevadas a cabo en 1984 no se pueden considerar bajo ningún concepto fórmulas de tránsito o de adaptación a los anteriores criterios expositivos, sino que desde sus comienzos Tecla Lumbreras tuvo muy claro las posibilidades de su proyecto y su puesta en práctica, extrayendo de los artistas su lado más lúdico y estimulante. Los ejemplos más claros son exposiciones del tipo del Templicon, Koberhegen, El estilo del relax, Los cuatro elementos, Marco's bar o Adornar el cuerpo/adornar el espacio.

La idea, planificación y el montaje de las exposiciones no contaban con la absoluta presencia de los artistas, sino que se implicaba a todo el colectivo del entorno de la galería y al público en general, haciéndoles partícipes de la propia obra y de su resultado final. Lo que hacía genuino el trabajo de Tecla Lumbreras era la predisposición a cualquier iniciativa que atisbara algún indicio que pudiera entenderse como artístico. Es el caso de exposiciones como Adornar el cuerpo/adornar el espacio; El estilo del relax, Los cuatro elementos o Bajondillo.

De su primer período al frente de la sala de exposiciones del COAM hay que destacar las exposiciones de Eva Lootz, Menchu Lamas, Nueve no vistos (colectiva con obras de Virginia Lorente, Manolo Criado, Melguizo, Enrique Queipo, Chema Lumbreras, Agustín Gallardo, Plácido Romero, Carlos Guevara y Benito Lozano); la obra de los tres artistas más importantes de la movida madrileña alternativa, Ceesepe, El Hortelano y Ouka Lele, Pintores Arquitectos con Guillermo Pérez

29 HERRERO E.: "Bola Barrionuevo, un pintor malagueño que se alegra de regresar a su casa, con su colección", Diario 16 de Málaga, 11-3-1990.

30 VV. AA.: 1980-2005, Veinticinco años de cultura en el Colegio de Arquitectos de Málaga, Málaga, Colegio de Arquitectos de Málaga, 2006. 


\section{Q: artículos Juan Carlos Martinez Manzano}

Villalta, Navarro Baldeweg, Campano y Gerardo Delgado.

La temporada de 1987-88 tiene un carácter más multidisciplinar, con exposiciones de pintura, arquitectura y escultura, haciéndose eco de otras disciplinas como el diseño, en la que contó con las exposiciones de Phillips Stark y Diego Santos; no faltando las exposiciones colectivas de artistas malagueños y afincados en Málaga como la llevada a cabo en mayo de 1987 con la muestra de Faustino Cuevas, Robert Harding, Margaret Harris, Herminia Hernández y Joaquín Gallego.

Tecla Lumbreras optó por esta fórmula en posteriores temporadas, sin olvidar su inquietud por las performance, siempre presente en cada una de sus etapas. Otra de las características de la temporada 88-89 fue la amplia aportación de artistas malagueños en sus diferentes generaciones, que supo combinar a la perfección, es el caso de la exposición de Pepe Seguiri como artista perteneciente a esa primera generación de finales de los 70 y Benito Lozano como artista representativo de finales de los 80 con el aliciente de mostrar el inicio de su etapa conceptual.

Tres artistas foráneos dentro de esta temporada; el artista checo Jiri Georg Dokopuil, uno de los artistas más importante de la generación Junger Wilder y del colectivo Mülheimer Freiheit, que tanto influye en la obra de Joaquín de Molina, Carlos Franco o Carlos Alcolea. Posteriormente con la muestra de Boshier, Tecla Lumbreras hace un homenaje a este polifacético artista estadounidense, que estuvo muy en contacto con el Pop Inglés, otro de los referentes de la "Nueva Figuración", y la obra del escultor, Frank Rebajes, distanciado del arte que se practicaba en los 80 , estando más comprometido con las vanguardias históricas.

El artista de moda en los 70 y buena parte de los 80, Pérez Villalta, empezaba a declinar en favor de una nueva generación de artistas formada entre otros por Dario Basso, Alejandro Gornemann, Darya Von Berner o Sergio Sanz, que de la mano de galeristas como Juana Mordó comienzan a despuntar en Madrid. A pesar de ello, en ciertos círculos, la obra de Pérez Villalta seguía en plena vigencia, siendo uno de esos lugares Málaga, donde no había dejado de exponer desde principios de los 80 , y el COAM no iba a ser menos. En esta temporada destacaron además las exposiciones colectivas: Colección de malagueños y Ven a verte con la participación del fotógrafo Pepe Ponce.

La temporada 89-90 cuenta con la presencia de un gran número de arquitectos y fotógrafos, disminuyendo la atención a los artistas locales. Se suceden exposiciones que muestran los trabajos de arquitectos como Venecia, Barragán, Piñón y Viaplana; de fotógrafos como Rosa Pedrero y Pepe Ponce; así como un gran número de colectivas y performance como Constructivistas, Concretos de Skene, Artistas de Cuenca, La ciudad velada, Ámbitos de la fotografía, Muebles diseñados por arquitectos, 6 escultores, Videos/instalaciones, El espejo negro.

Los artistas con los que colaboró Tecla Lumbreras en este periodo de finales de los 80 fueron muchos más de los que se pueden contabilizar en el catálogo resumen de la muestra antológica llevada a cabo en la sala de exposiciones del Palacio 
Episcopal, ya que muchos de ellos colaboraron de modo desinteresado, montando exposiciones, aportando ideas o prestándose a cualquier tarea relacionada con las variadas manifestaciones que la dirección de la sala ponía en marcha. Muchas de las exposiciones partieron de iniciativas propias de los artistas, íntimamente ligados con la sala de exposiciones, lo que desembocó en una integración y adaptación de las ideas más dispares dirigidas no solo a realizar una tarea expositiva sino una forma de colaboración entre los artistas y la galería, que se transformó en una especie de laboratorio de ideas, con un público entregado a las nuevas iniciativas de la galería. Esta forma de actuación generaba, se encuentra en montajes creados a finales de los 80 , destacando: Bajondillo/Tótem para una playa, Marco's bar, Adornar el cuerpo/adorar el espacio, Los cuatro elementos y El estilo del relax. En todas ellas hay una traslación de elementos, materiales, personajes y ambientes en los que se engloba el propio público, todo ello bajo el telón de la ironía y el debate sobre la postmodernidad que es lo que fluye en todo el discurso crítico de los 80 . Un planteamiento visual llevado a la práctica artística con manifestaciones lúdicas, juegos efímeros, falsos laberintos, recreaciones animadas, efectos visuales, escenificaciones improvisadas, que van reinventando el espacio constantemente, como el inicio de la obra que está por concluir.

Con respecto a la acogida que tuvo la generación de artistas figurativos de finales de los 70 y principio de los 80 , estuvo sujeta a una frágil línea expositiva repartida entre 1984 y 1990. Tuvieron un papel destacado Gabriel Padilla, Pepe Seguiri, Plácido Romero, Isabel Garnelo, Chema Tato, Joaquín de Molina, José Luís Díaz Pardo, Chema Lumbreras y José Luis Bola. De los pocos que en el periodo 8489 no tuvieron una exposición individual fue Carlos Durán, lo que no quiere decir que estuviera excluido de todos los proyectos de la galería, ya que interviene en la muestra dedicada a la máquina arquitectónica, el Templicón, de Juan Antonio Ramírez y dentro del ciclo El diseño en Málaga de mayo de 1985. Pero los hubieron con los que no se contó de ninguna manera hasta 1992, es el caso de Daniel Muriel.

Con respecto a las colectivas de artistas malagueños fueron bastantes las producidas. De 1985 es la exposición Nueve no vistos. Pintores en Málaga, en la que participó Manolo Criado, Agustín Gallardo, José Carlos Guevara, Virginia Lorente, Benito Lozano, Chema Lumbreras, José Melguizo, Enrique Queipo y Plácido Romero. En abril de 1986 tuvo lugar otra colectiva con Pepe Castellano, Juan Tendor y Pérez Sánchez. De octubre de ese mismo año es la exposición colectiva de Javier Hidalgo, Antonio Villalobos, Encarni Lozano y Alejandro Romero. De noviembre de 1986 es el Salón de los Rechazados, con Diego Santos, APS, Pepe Castellano, Isabel Garnelo, Chema Lumbreras, José Luis Santana y Juan Tentor. De mayo de 1988 es la colectiva de Faustino Cuevas, Joaquín Gallego, Margaret Harris, Herminio Hernández, Concha Mamely y Robert Harding. De abril de 1989 será la colectiva más numerosa con obras de Antonia Barba, María Jesús Camacho, Joaquín Ivars, Carmen López, Lope Martínez Alario, Titi Pedroche, Carlos Brotons, José Ma Olid, Francisca Serón y Ángel Horcajadas. En 1990 la colectiva llevada a 
cabo en el mes de abril presentaba una nueva hornada de artistas malagueños, con Bisbé, Negro Macho, Ángela Ache y Dolores Lamamié, de los cuales Bisbé es el único malagueño, siendo ésta una de sus primeras exposiciones.

La comisión de cultura de la sala de exposiciones del COAM contó en numerosa ocasiones con la participación del colectivo de artistas Agustín Parejo School, destacando la muestra que tuvo lugar en febrero de 1985, De côté de I'URSS ${ }^{1}$, homenaje a la vanguardia cubo-futurista rusa, donde hicieron alarde de los ideogramas más característicos del movimiento soviético. La exposición fue algo más que una simple aproximación a las convicciones políticas e ideológicas que caracterizaron a muchos de los colectivos artísticos que se crearon desde finales de los 70 y principio de los $80^{32}$, fue además un derroche de imaginación donde se combinaron multitud de disciplinas artísticas, desde la pintura pasando por la fotografía, el video, el post-art, el copy art, la cartelería etc. Una versátil demostración de la capacidad imaginativa y creativa del colectivo, acompañada por la música del grupo U.H.P33. De côte de I'URSS fue además una revista diseñada de forma artesanal, según acostumbraba a realizar el colectivo, cuyas manifestaciones artísticas se hacían acompañar de toda una parafernalia de objetos, videos, cartelería, camisetas, fotografías... alusivos a sus creaciones, que en la mayoría de las ocasiones partían de una simple frase, una palabra, un eslogan o una poesía.

Las demás participaciones de los APS en el COAM durante los 80 tuvieron lugar en mayo de 1985 en una colectiva ya citada, Diseño en Málaga, donde a cada uno de los artistas convocado se le proporcionaba una costurera para que diseñaran y realizasen una línea de moda. En octubre de 1985 junto con Manolo Castro, Gabriel Padilla, Diego Santos, Pepe Seguiri y Alfonso Serrano, participaron en Arte en tres dimensiones. En febrero de 1986 presentan en la sala de exposiciones la revista Euskadi da, una publicación artesanal al estilo de Absolument mondernier ${ }^{34}$, siendo todo un manifiesto de intenciones que además se presenta a partir de diferentes alternativas plásticas como una pintada callejera, un video denominado Málaga Euskadi da y una cinta musical del grupo U.H.P, cantada en euskera. En noviembre de 1986 aparecen en la colectiva El salón de los rechazados, y en noviembre de 1990 participan junto a Encarni Lozano en la exposición Berlín-Berlín, que parte de una idea de Jorge Dragón para conmemorar el derribo del muro de Berlín.

La galería de Carmen de Julián, abierta al público en diciembre de 1987 mos-

31 Vid. "El colectivo Agustín Parejo School presenta Côte de I"URSS", Diario Sur, Málaga, 7-11-85, cfr. PUJALS E.: "El arte de la fuga: modo de producción artística colectiva en España 1980/2000", www.arteleku, pág.156.

32 Estos colectivos, inmediatamente anteriores a la muerte de Franco y que se prolongan a través de la transición, llevan a cabo un "activismo" cultural encaminado a una revolución obrera. Los más destacados fueron La familia Lavapies, La Fiambrera Obrera, y en los 80, con el consumado desencanto del advenimiento del proceso democrático, se crean colectivos como Strujembank, Agustín Parejo School y Preiswert.

33 Op. cit. pág. 154.

34 Absolumnent Mondernier es uno de los primeros fazinne de producción artesanal creado por el colectivo a principios de los 80, al que siguieron Mal a riem poem, Du côte I'URSS y Euskadi da. 
trará una línea ambigua que queda patente en las tres colectivas llevadas a cabo entre junio de 1988 y febrero de 1989, con artistas muy dispares que contaminan constantemente el discurso artístico que pretenden conformar. La primera de estas tres colectivas se lleva en junio de 1988 con obras de Rafael Alvarado, E. Fernández, A. González, Sebastián del Campo, S. Vivas y Melguizo. La segunda es un resumen de las exposiciones llevadas a cabo con anterioridad, con Claudio Díaz, Barbadillo, Pepa Caballero y Paco Molina. Quizás la más interesante de las tres exposiciones colectivas sea la llevada a cabo en febrero de 1989 donde se dieron cita obras de Luis Gordillo, Carlos Durán, Pepe Seguiri, Pérez Villalta, Lamazares, Peláez, Patiño, Menchu Lamas, Brickmann, Peinado y Lindell. Esta última colectiva resume la trayectoria de Carmen de Julián; la mezcla de estilos, tendencias y generaciones se hacen más que evidentes en una selección que no tiene razón de ser sino mantener abiertos todos los frentes de aquellos artistas más significativos y "vendibles" del momento, desde Gordillo pasando por Pérez Villalta y terminando en la obra de Menchu Lamas, Carlos Durán y Seguiri, haciendo un recorrido sinóptico de las tendencias figurativas más innovadoras de las últimas dos décadas. Si la obra de Patiño y Lamazares -artistas vinculados al Grupo Atlántica junto a Menchu Lamas- comenzaba a separarse del fuerte discurso que impone la nueva figuración de los 80 , la introducción de Brickmann, Peinado y Lindell no deja muy claro a que criterio responde.

En diciembre de 1988 expone de nuevo un artista sevillano, Marina Díaz, perteneciente al círculo de Paco Molina y Sebastián del Campo, autores muy relacionados con el ambiente realista sevillano de los 70 -tal y como comenté anteriormenteacabando 1988 con una muestra del artista catalán Joan Brossa. En 1990 destacar la exposición de Carlos Durán.

Lo que queda claro es que en 1987 el colectivo Palmo desaparece, Pedro Pizarro aun continúa en Alhaurín el Grande y la galería Harras también cierra sus puertas. Carmen de Julián, junto a la sala del COAM y la sala Bacardí, amortiguan, en cierto modo, la sensación de vacío que se percibe dentro del panorama expositivo de la ciudad, que tardará algunos años en recuperar su vigor con la apertura de la galería Pizarro, la nueva iniciativa expositiva del CAA, la llegada de Mariluz Reguero a la Sociedad Económica "Amigos del País" y el impulso de la universidad a las artes plásticas.

La sala de la Diputación Provincial inicia la segunda mitad de la década con la etapa García Cubo, que persiste en exponer a los artistas de la nueva figuración madrileña de comienzo de los 70, con la obra de Chema Cobos. En febrero de 1985, llega una emotiva exposición de Joaquín de Molina ${ }^{35}$, la última realizada por este artista meses antes de su fallecimiento, haciendo un recorrido antológico por sus diferentes etapas, en especial las de Madrid, Barcelona, Emme y Berlín.

La gran apuesta expositiva de García Cubo llegaría en abril de 1985 con la organización de una muestra en la plaza de toros de La Malagueta denominada

35 MAYORGA J.: "Una vigorosa muestra de la pintura berlinesa de Joaquín de Molina en la Diputación", Diario Sur, Málaga, 28-2-1983. 
Galicia en el ruedo contando con la colaboración de un total de 15 artistas entre los que se encontraban: Freixane, Carbajo, Correa Correidora, Carlos Crego, Huete, Menchu Lamas, Martínez de la Colina, Din Matamoro, Ana Manzoi, Moldes, Morquecho, Antón Patiño, Manolo Rubial y Jesús Vázquez; los escultores: Ballallo, Borrajo, Pestena y Leiro. Gran parte de estos artistas pertenecen a la generación del "Movimiento Atlántico", adscritos a la nueva figuración gallega ${ }^{36}$, que tuvo su punto culminante en la exposición llevada a cabo en 1985, denominada Nueva figuración gallega, una muestra itinerante que recorrió gran parte del continente americano.

Patricia Gadea otra de las pintoras que se desenvuelven dentro de la movida de los 80 expone en junio de 1985. Protagonista en primera persona junto a Juan Maldonado, José Ugalde, Carlos Forns Bada, Sufrido Martín Beige y Antón Llamazares de la segunda generación de pintores figurativos madrileños y que exponían asiduamente en la galería Ovidio de Madrid. En julio de 1985 expone el sevillano Juan $\mathrm{F}$. Lacomba pintor tangencial con respecto a la figuración madrileña, que presentó la muestra Picacho-fósil-Carmona/85.

En noviembre de 1985 vuelve a fijarse en la figuración malagueña con una exposición de José Luís Bola Barrionuevo ${ }^{37}$, después de varios años sin exponer en la ciudad. En febrero de 1986 se realiza una muestra conjunta con el Museo de Bellas Artes de la obra de Francisco Peinado, volviendo a la línea de artistas nacido en los 50 con la obra del sevillano José María Bermejo en marzo de 1986.

Con la exposición de Bermejo se da por finalizada la etapa García Cubo y se inicia la etapa de Sebastián Camps. En una entrevista publicada en octubre de $1986^{38}$ Sebastián Camps manifiesta su predilección por los pintores que iban a contracorriente, sin ataduras a estilos ni tendencias, eligiendo para sus primeras exposiciones a Adriá Pina y Jesús Conde. Poco tiempo duraron las buenas intenciones de Sebastián Camps ya que a continuación acude a Rafael Alvarado, lo que será una muestra de la inconsistencia de su discurso expositivo.

En noviembre de 1986 se presenta la exposición Jesús Conde, carente de recursos estilísticos y denotando una simplicidad manifiesta en consonancia con la etapa de Sebastián Camps. En diciembre de 1986 presenta a una perfecta desconocida, Teresa Pla, iniciando 1987 con una muestra de Francisco Peinado a la que le sigue otro pintor malagueño, Bernardo Roquero, formado artísticamente en Sevilla y profesor de la Escuela de Arte y Diseño de Málaga, y del cual no se puede reseñar ninguna exposición de interés. En marzo y abril dos exposiciones de cierto interés, la de Chema Tato en marzo y la de el artista jienense David Padilla.

Con estas dos exposiciones parecía que Sebastián Camps retomaba el rumbo de las nuevas generaciones pero todo quedó en un espejismo, ya que a finales de marzo se presenta una muestra homenaje a Van Gogh a la que le sigue Titi Pedroche

36 Vid. "De la nueva figuración atlántica", La Voz de Galicia", A Coruña, 11-5-2007.

37 MAYORGA J.: "Diálogo entre fondo y figura en la obra de Bola", Diario Sur, Málaga, 2-11-1985.

38 Vid. Diario Sur, 20-10-86. 
en abril de 1986, con una obra ya conocida en Málaga. La exposición de Natali Pinazo en mayo de 1986 y una antológica dedicada a la tauromaquia picasiana da muestra del estancamiento de esta sala durante el periodo de Sebastián Camps. Con las exposiciones de Natali y Pedroche parece recurrirse a un enfriamiento artístico después del predominio de la nueva figuración, con dos autores poco señeros para estos propósitos, consiguiendo un contrapunto con respecto a las exposiciones de Tato y Padilla, aunque no creo que este fuera el propósito último de Sebastián Camps, más bien sería aunar exposiciones de artistas que el público identificara.

La característica de estos últimos años será los periodos de inactividad, con el siguiente bagaje: José Luis Rodríguez expuso en diciembre de 1987 una muestra de fotografía, Pascual Torres en diciembre de 1987, en febrero de 1988 Antonio Jiménez, en abril de 1988 expone Sebastián Navas, una exposición colectiva sobre los derechos humanos con la participación de Plácido Romero, Paco Aguilar, Gabriel Padilla, Carlos Durán, Alfonso Serrano, Chema Lumbreras, Juan Tendor, Manolo Criado, Enrique Queipo, Rosa Pedrero, Juan Carlos Guevara y Paco Rojas; Enrique Queipo en enero de 1989 y de nuevo Carlos Durán en febrero de 1989.

La última de las etapas con la que la sala de arte de la Diputación Provincial cierra los 80 será la de Joaquín Abenza, que en febrero de 1989 toma el relevo de Sebastián Camps al frente de la sala de la Diputación Provincial con una exposición de Faustino Cuevas en marzo de 1989 y en julio de 1989 de Chema Lumbreras, otro de los jóvenes artistas pertenecientes a la segunda generación figurativa malagueña, con los que se acaba la década.

El hecho de que un gran número de espacios expositivos nacidos durante la primera mitad de la década de los años 80 hayan desaparecido a mediados de 1984 no es nada excepcional, ya que los espacios expositivos de Málaga tienen una duración media de poco más de dos años, con la salvedad del Colectivo Palmo, Terral y los espacios institucionales activos en el segundo lustro de los 80 . El resto han desaparecido, dando paso a otros que tampoco aportaron demasiado al panorama artístico de la ciudad. Los que han desaparecido son: 7/10, Harras, Vilches, Picasso, Miguel Ángel, Arkadia, Arcorac, Pámpano, La Herradura de Oro, Café-arte, Yunque y Casablanca. Cerraron sus puertas entre 1983 y 1985. Lo positivo de estos cierres prematuros, es el nacimiento de otros espacios expositivos que abren durante esta segunda mitad, los más destacados serán: la Sala de Arte Bacardi, La Maisón, Benedito, Porticus, Café-teatro, sala Moreno Villa, Goya, La Medina, Arribabar, Fórmula Ocio, galería Ugalde, sala de exposiciones del Instituto Vicente Espinel y la sala de arte ADE. Otros se han remozado tanto en sus instalaciones como en su línea expositiva, es el caso de la sala de arte de la CAA.

Terral, a partir de 1984 muestra una línea de apoyo a las jóvenes generaciones, aunque con menos intensidad a partir del último tercio de la década, exponen artistas consagrados que van alternando con los recitales de poesía de escritores noveles. Dentro del periodo que va de 1986 a 1990 las exposiciones más destacadas son las de 
Adolfo Santarem, con el título Recién pintado, realizada en marzo de 1986; a la que le siguió una exposición de Antonio Barba en junio y otra de Daniel Muriel en octubre.

En febrero de 1989 se lleva a cabo una colectiva que rompe con toda la dinámica de Terral desde su fundación, una exposición denominada Realismo con obras de Blanca Sánchez, Barberán, Fermín Durante, Robles Muñoz, Molero y Ruiz Blanco; apegados a la tradición de la estampa de corte localista. En marzo de 1989 Terral recobra el rumbo con la primera exposición individual del malagueño Joaquín Ivars; inmerso aun en su primera etapa, cercana al minimalismo e inmediatamente anterior a su paso al conceptualismo de los 90. En marzo de 1989 una colectiva de mujeres Profumo di donna que contó con la participación de Isabel Garnelo, LilianaMauro Coger, Encarni Lozano, Pilar Chamorro, Concha Mameli y Titi Pedroche.

La nueva línea expositiva del C.A.A. propuesta por Alfonso Canales a mediados de 1988, abandona la condición de espacio abierto que tuvo en los inicios de los 80 , para convertirse en una sala que fuera una alternativa al discurso de las nuevas galerías ${ }^{39}$. Este cambio se puede deber a que los espacios expositivos intentan elevar su nivel en consonancia con el movimiento que empieza a generarse en torno a las galerías ya reseñadas de Pedro Pizarro y Tecla Lumbreras, además del atractivo añadido del auge de los medios escritos y la proliferación de críticos de arte. Con anterioridad la sala de arte de la entidad bancaria seguía el modelo de la primera mitad de la década con exposiciones de artistas desconocidos alternando con jóvenes que empezaban a tener cierta proyección, como Lope Martínez Alario que expone en mayo de 1985. A Lope Alario le sigue una serie de nombres desconocidos dentro del ámbito artístico malagueño del momento, con exposiciones de Antonio Quero en febrero de 1986 y Mercedes Martín, en marzo del 86. En junio del 86 expone Pedro Somera, artista perteneciente a la generación de finales de los 60 con una trayectoria claramente descendente, para continuar con José Fernández Ballestero en julio de 1986, Francisco Navas en septiembre del 86, Alfonso Martínez en enero de 1988, Fernando Wilson, en febrero de 1988, Manuel Carmona, en abril del 88, Carlos Barceló en junio de 1988 y Carranza Marmolejo también en junio de 1988. A partir de este momento la sala se somete a una intensa reforma, ampliando sus instalaciones y dotándola de mejores medios. Este cambio de aspecto también se dejó sentir, como ya he comentado, en la línea expositiva a seguir. El primer artista que inaugura este nuevo periodo es el pintor sueco Bengt Lindström en octubre de 1988, al que le sigue Stefan en diciembre de 1988 y Héctor Carrión en enero de 1989, tres artistas de la generación de finales de los 50 y principios de los 60 que nada aportan al panorama de los ochenta, pero que intensifican la labor de la sala dentro del organigrama expositivo. A continuación la obra gráfica de Montijano en febrero de 1989; una colectiva de 30 artistas en abril de 1989 y una peculiar muestra de la obra pictórica de Julio Caro Baroja.

Para terminar este amplio recorrido por los espacios expositivos de la Málaga de los 80 , hacer mención de la sala de exposiciones del Cortijo Bacardi, situada en

39 Vid. "Nueva etapa del C.A.A.", El Observador, n 10, Málaga, 1988. 


\section{Q: artículos Contextualización y promoción artística...}

uno de los polígonos industriales del cordón metropolitano malagueño. Desarrolla su actividad ininterrumpidamente desde 1986 hasta 1999, siendo otro ejemplo de los cambios que se están produciendo en el ámbito artístico malagueño, con una primera etapa titubeante que va desde 1986 a 1988, donde la nota predominante es la alternancia de artistas nacionales con internacionales sin demasiada relevancia. La segunda etapa se inicia acabando la década y enfocada hacia los artistas malagueños surgidos de la nueva figuración, destacando la colectiva realizada en abril de 1989; con obras de Brickmann, Faustino Cuevas, Díaz Pardo, Carlos Durán, Antonio Jiménez, Chema Lumbreras, Gabriel Padilla, Francisco Peinado, Plácido Romero, Pepe Seguiri y Fernando Teixidor. Sin dejar de ser la típica exposición colectiva que muestra las diferentes generaciones a modo de abanico panorámico, da entrada a nuevas promesas como los jóvenes Faustino Cuevas, Chema Lumbreras y Plácido Romero. La clave de esta exposición es que se hace bajo una directriz determinada inserta en su enunciado: Bajo el signo de Eros, por lo que se vuelve a apreciar un discurso expositivo, una vía de escape a las exposiciones contenedores, además de contar con la peculiaridad de que en esta ocasión todos los artistas son malagueños. Este tipo de exposiciones que en Málaga iniciara, allá por 1982, García Cubo en el Ateneo de Málaga con la macro-exposición denominada 1.000 .000 de pavos o Artistas en el Ruedo, siendo director de la sala de la Diputación Provincial, se generalizaron posteriormente con Pedro Pizarro en exposiciones como las ya comentadas San Sebastián o Co-mercia y con Tecla Lumbreras, la que más se prodigó, así como el colectivo el Palmo con las exposiciones El disco de tu vida o El hombre y el salario. Estas exposiciones temáticas fueron para el espectador un modo didáctico de entender las diferentes tendencias que se aunaban en la plástica malagueña, así como un aliciente más lúdico para los artistas que se prestaban a este tipo de muestras. 
